\title{
Internet of Things Technology and Its Application in Power System Communication
}

\author{
Zhang Yong, Xie Lianke, Zang Yuwei, Liu Hui, Wang Kun \\ State Grid Shandong Electric Power Research Institute, Shandong Jinan, 250002
}

Keywords: Internet of things technology; power system communication; application

\begin{abstract}
Internet of things technology is a new network technology which develops fast. In the process of application, it can realize the connection between goods and network and give vitality and energy to goods. With the constant optimization, update and development of the Internet of things technology, it has been widely used in the power system communication at this stage. The applications of the Internet of things technology in the process of power system communication network technology are mainly in the aspects of emergency communications, distribution network communications, smart grid, social management service and so on, which promotes the optimization of the quality of power system communication and the improvement of management level. In order to analyze the physical network technology and its application mode and approach in the process of power system communication, the author analyzes and explores the essence and the structure of Internet of things and the Internet of things technology.
\end{abstract}

\section{Introduction}

Power communication and information system in the process of power system communication at current stage has fully realized the coverage of power plant, grid, substation, power supply bureau, power supply station and so on. The power system communication, according to the needs of different business, establishes the corresponding comprehensive data network, the transmission network, the dispatching data network and so on ${ }^{[1]}$. To a certain extent, it promotes the realization of the interconnection of communication network at various levels. The network are extended and expanded to all objects of distribution network and life. It realizes the communication among objects through the implementation of the Internet of things technology and function ${ }^{[2]}$. The main purpose of this study is to analyze the Internet of things technology and its application mode and approach in the process of power system communication. Before analyzing the application of the Internet of things technology in power communication system, the author first accordingly and specifically analyzes the essence and the structure of Internet of things and the Internet of things technology.

\section{Analysis of the Essence and the Structure of Internet of Things}

The Internet of things mainly refers that all goods rely on RFID and other information sensing devices to make interconnection with the Internet, so as to achieve the purpose of intelligent identification and management of goods. The emergence of the Internet of things completely breaks the concept of the mutual separate status in the traditional sense of the IT infrastructure and physical infrastructure. In the development process of the Internet of things era, it can integrate, optimize and correlate the relationships among the architecture or the facility made by reinforced concrete and cable, the electronic chip and the network. It can establish the unified modernization infrastructure and create a new platform, which can integrate the social life, economic management, production, sales and other activities together. It will improve the quality of people's life and production efficiency and promote human progress and the rapid development ${ }^{[3]}$.

The network of Internet of things is mainly composed of access layer, application control layer, user layer, bearer network, application collection control layer and so on. Among them, the computer network and the communication network constitute the bearer network together. The 
access layer is mainly composed of base station nodes and access gateway and terminal nodes mainly include control modules and diversified collection module. The access layer collects information and controls networking by terminal nodes and can timely and effectively transfer and convey information through the peripheral nodes; the application control layer is mainly composed of the database server and the application server and is of certain functions of conversing, collecting and analyzing data. It can realize the adaptation and practice of user layer. The bearer network is communication network and is responsible for the function of information communication of the access layer of Internet of things and the application control layer; the user layer mainly refers to providing interface of the application interface of Internet of things for user. It mainly includes client, mobile phone, computer, sensor and all kinds of user equipment ${ }^{[4]}$; the bearer network also refers to communication network, such as mobile communication network and Internet and so on. It is of the significance of the information communication function of the access layer of Internet of things and the application control layer. The bearer network mainly includes loop network, tree network, end-to-end network, interactive network and so on. Its form is divided into two kinds which are wired networking and wireless networking. The wired networking mainly includes Internet data line, voice communication cable and optical fiber. The wireless networking mainly includes the interoperability network of worldwide microwave access, the local loop network of multi-carrier wireless information and the cluster network and so on.

\section{Analysis of Internet of Things Technical and physical network technology and its application mode in the process of power system communication}

\subsection{Analysis of Internet of Things Technical}

The Internet of things mainly contains four key application technologies: intelligent technology to think about things, sensor network technology to perceive things, RFID technology to identify things and nanotechnology to miniature things. RFID technology to identify things automatically identifies the target object and obtains its basic data and information. It can conduct a kind of non-contact and automatic recognition technology in a bad environment. It is mainly composed of logo (composed by chip and coupling element), reader (the equipment to read the identification information) and antenna (RF signal transmission for identification and reader). The intelligent technology to think about things makes objects be of certain intelligence mainly through the way of implanting the intelligent system into objects and automatically processes by computer. The research domain of intelligent technology mainly aims at the principle of realization of computer intelligent, intelligent control technology and system, artificial intelligence and intelligent signal processing and so on. The RFID technology to identify things mainly studies the related security and privacy issues, the anti-collision and conflict-prevention issues and the RFID antenna and so on. The sensor network technology to perceive of things gathers the network communication system of wireless sensor network with the distributed information acquisition, information transmission and information processing technology as a whole. It is of the characteristics of low cost, miniaturization, flexibility and low power consumption and so on, which is suitable for the moving target. The basic component elements of sensor network nodes are the sensing unit composed of sensor and analog-digital conversion function, the processing unit composed of memory, CPU, embedded operating system ${ }^{[5]}$ and the communication unit and power source composed of wireless communication module and so on. The research on sensor network technology mainly aims at the research on structure and underlying protocol of sensor network technology, the research on intelligent sensor of network node, the detection and control of sensor network itself, the security of sensor network, the advanced test technology, network measurement and control and so on. The nanotechnology to miniature things mainly studies the property and application of materials of small-size structure, including Nano chemistry, Nano physics, Nano biology, Nano electronics, Nano mechanics, Nano processing and Nano materials and so on.. 


\subsection{The application mode of Internet of things in the process of power system communication}

\subsubsection{The application of Internet of things in the process of power system emergency communication}

Emergency communication may occur at any time in the process of power system communication. It is of uncertainty of time and place, increasing the difficulty of the connection between the command center and the accident site to some extent. In the traditional process, in general, the rescue personnel conduct check and collection according to the field information after rushing to the scene and send the field information back to the command center in the way of file or video with the corresponding audio and video and so on, which wastes the rescue time to a certain extent. With the appearance of the Internet of things technology, it improves the work efficiency of the emergency communication and provides great convenience and saves a lot of time for the implementation and the organization of corresponding work of command center and dispatch center. Detect the state information of grid and operation condition of all kinds of equipment through the Internet of things networking intelligently. Once detecting the emergency, the Internet of things can send the accurate position of accident scene and specific condition of on-site accident in the form of timely sending data information to the command center ${ }^{[6]}$, which is convenient for emergency staff to prepare the corresponding equipment and appliances and so on in advance. They can immediately carry out the work of replacement and repair after reaching the scene, which improves the work efficiency of dealing with emergency work, reduces the workload of the emergency staff and saves a lot of time.

\subsubsection{The Application of Internet of Things in the Process of Distribution Network Communication of Power System}

The distribution network is of characteristics of many voltage grades is more and more, complex structure, much power distribution equipment, wide distribution, frequent phenomenon of dynamic changes, many branch lines. According to relevant requirements of the level of distribution network automation, the whole network communication is mainly divided into primary station of distribution network, communication substation, district-transfer substation layer and other aspects. The primary station of distribution network mainly refers to the communication role of communication substation and primary station of distribution. According to relevant technical requirements, the data bearer of primary station of distribution network should be in dispatching data network; the communication substation layer mainly refers to the communication from distribution network terminal to the communication substation layer. The communication substation is usually set in $110 \mathrm{KV}$ or $220 \mathrm{KV}$ substation; the district-transfer substation layer is mainly the communication level of district-transfer substation and primary station of distribution. The amount of the data of district-transfer substation layer is larger. It is also stored in data network. The distribution network communication at the present stage cannot use single communication method and is achieved mainly relying on carrier communication, optical fiber communication and leased wireless broadband technology. Because the distribution network has greatly changed, the optical fiber private network is of certain difficulty in construction. The modification of it is not convenient and the construction cost is high; the safety of wireless public network and carrier communication is poor. Therefore, effective Internet of things technology is particularly important. The Internet of things can solve the problem of communication between the primary stations of distribution network mainly through connecting the Internet of things with the equipment and parts of distribution network and finishing the corresponding task of distribution communication. The Internet of things can solve the problems of large numbers and larger changes of distribution terminal and so on ${ }^{[7]}$.

\subsubsection{The Application of Internet of Things Technology in Smart Grid of Power System}

The smart grid is a new kind of grid form with high safety degree and flexible use and can meet the development needs of low carbon environmental protection currently advocated in society to a large extent. It can combine communication technology, information technology, sensor 
measurement technology, control technology, and decision-support technology and so on to realize the efficient and secure operating state. The application of smart grid largely conforms to the call for environmental protection in the current society and belongs to the emerging technology of environmental protection. Applying the Internet of things technology in smart grid is mainly for effectively complementing and enhancing measurement technology, communication technology, control technology, monitoring equipment state, remote meter-reading technology, energy-saving control technology, grid load energy-saving control technology, transmission line tower, icing monitoring technology, temperature monitoring technology, related accident accurate positioning and tracking technology and other technologies and means ${ }^{[8]}$. It can collect all kinds of communication data in a certain extent. With the help of integration of Internet of things and collection all kinds of underlying information, it can improve management efficiency and ability of dealing with emergency in a certain extent, which effectively prevents the happening of power accidents and improves the processing efficiency after accidents to reduce the damage caused by accidents.

\section{Conclusions}

The implementation of corresponding Internet of things technology in the operation process of power communication mainly refers that through applying intelligent technology to think about things, sensor network technology to perceive things, RFID technology to identify things, nanotechnology to miniature things and other technologies of Internet of things, implement the corresponding application applied in the process of emergency communication of power system of Internet of things, the application applied in the process of distribution network communication of power system of Internet of things technology, the application applied in smart grid of power system of Internet of things technology and other ways. Improve the management level and management efficiency of power system communication to the maximum extent. Prevent the happening of all kinds of underlying power accidents. Accelerate the processing speed of all kinds of underlying power accident after happening. In the process of the actual use of Internet of things technology, there are still some problems restricting the popularization and the development of Internet of things technology, so in the process of daily work we should reinforce the research and promotion of Internet of things to realize common development of the Internet of things and the power system.

\section{References}

[1] Xia Fei, Huang Xiaobo. Analysis on Application of Internet of Things Technology in Power System Communication [J]. China Science and Technology Expo, 2014, 11(47): 255-255.

[2] Xue Yanxia, Ren Sheyi. Internet of Things Technology and Its Application in Power System Communication [J]. Construction Engineering Technology and Design, 2015, 07(27).

[3] Huang Shaohua. Rely on Internet of Things Technology, Improve the Supporting Capacity of Power Communication to Smart Grid [J]. China Science and Technology Inestment, 2016, 15(32).

[4] Chai Yukun. Application of Internet of Things Technology in Smart Grid [J]. China Electric Industry: Technical Edition, 2014, 24(2): 57-62.

[5] Su Qin, Sui Mingchao, Yang Xulong. Application of Internet of Things Technology in Management of Substation Equipment [J]. China Science and Technology Expo, 2015, 21(28): 234-234.

[6] Li Hongwei, Liu Yue, Yu Gaobo. Research on Application of Internet of Things Technology in Power Communication [J]. Digital communication world, 2016, 32(9).

[7] Xu Yiming. Research on Communication Technology for Internet of Things of Power [D]. North China Electric Power University (Beijing), North China Electric Power University, 2016.

[8] Niu Xiao, Tian Lina. Exploration of Integrated Development of Information and Communication Technology of Power System [J]. Goods and Quality, 2017, 12(3). 